\title{
Characterization of Photophysical Properties of the
}

\section{Photoactivatable Fluorescent Proteins for Superresolution}

\section{Microscopy}

Antai Tao, Rongjing Zhang, Junhua Yuan*

Hefei National Laboratory for Physical Sciences at the Microscale and Department of

Physics, University of Science and Technology of China, Hefei, Anhui 230026, China

\section{Supporting information}

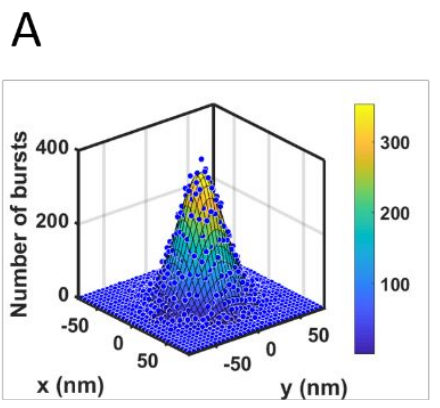

B

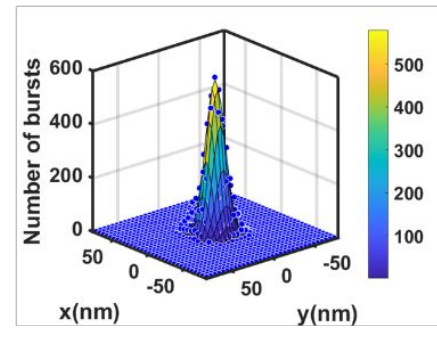

C

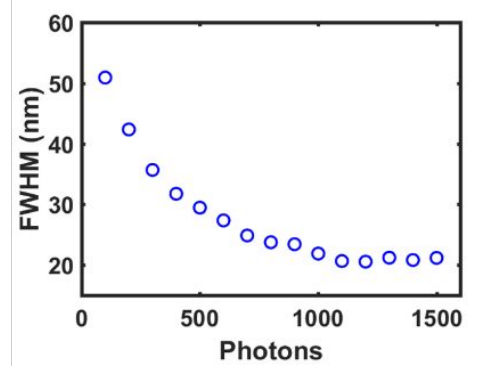

Fig. S1. Resolution vary with the number of photons. (A) The two-dimensional histogram of burst ( $\sim 100$ photons) positions and Gaussian fitting of the histogram. (B) The two-dimensional histogram of burst ( $\sim 1500$ photons) positions and Gaussian fitting of the histogram. (C) Relationship between the number of photons per burst and FWHM of the Gaussian fitting. The limit of FWHM was $\sim 20 \mathrm{~nm}$. 


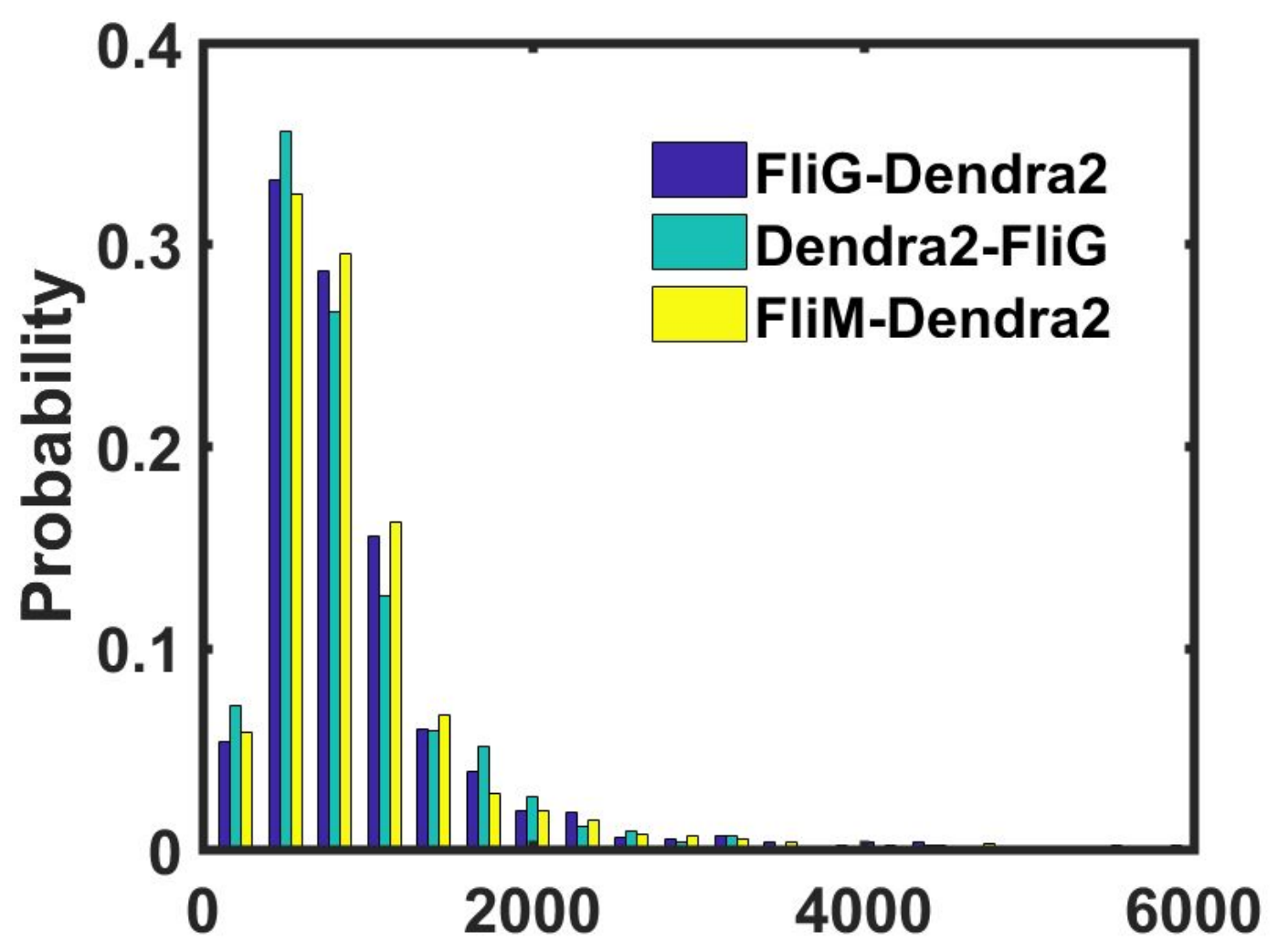

Fig. S2. Histogram of the number of photons emitted in each burst from Dendra2 molecules in fixed cells. 